\title{
Development and evaluation of a photographic atlas as a tool for dietary assessment studies in Middle East cultures
}

\author{
Maya Tueni ${ }^{1,2, *}$, Afifee Mounayar ${ }^{1}$ and Ines Birlouez-Aragon ${ }^{2,3}$ \\ 'Department of Nutrition and Dietetics, Faculty of Science II, Lebanese University, Fanar, Lebanon: \\ ${ }^{2}$ AgroParisTech, Paris, France: ${ }^{3}$ Spectralys Innovation Biocitech, Romainville, France
}

Submitted 9 September 2010: Accepted 6 January 2012: First published online 10 February 2012

\begin{abstract}
Objective: To assess the errors between estimates of amounts based on photographs and actual quantities of food presented to adults or eaten on the previous day. Design: A photographic atlas was constructed by taking digital photographs of three pre-weighed portion sizes of 212 traditional Lebanese dishes. In a first approach, ten portions of real pre-weighed foods were assessed using the photographic atlas. In a second approach, the participants weighed all foods consumed at one meal and recalled the amount of food that was eaten the previous day using the photographic atlas. Differences between actual quantities and estimation were assessed using a signed rank test $(P<0 \cdot 05)$. Spearman's correlation coefficients and bias (Bland-Altman plot) between the methods were calculated.

Setting: Lebanese university canteen and Lebanese homes.

Subjects: Forty adults (twenty males, twenty females) completed the first protocol and fifty adults (twenty-five males, twenty-five females) completed the second protocol; all were volunteers aged 21 to 62 years.

Results: Mean differences between actual and estimated portion sizes by photographs were between $-13 \cdot 1 \%$ and $+24 \cdot 5 \%$ when pre-weighed foods were presented, and between $-10 \cdot 4 \%$ and $+3 \cdot 8 \%$ when foods were consumed the day before. No significant differences were found between actual and estimated portion sizes except for three dishes (loubieh bil zeit, tabbouleh and yachnet bazella). Bland-Altman plots showed good agreement for all dishes with a negligible bias of $-0 \cdot 2 \mathrm{~g}$ between estimated and presented portions, and $-6 \cdot 3 \mathrm{~g}$ between estimated and eaten portions.

Conclusions: In the Lebanese diet, food photographs seem a reliable tool for quantification of food portion sizes.
\end{abstract}

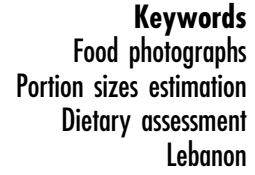

In dietary epidemiological surveys, portion sizes of commonly consumed foods are often roughly estimated due to high difficulty in remembering and evaluating them properly ${ }^{(1)}$.

Two methods have been used to quantify food portion size: (i) those in which foods are weighed directly; and (ii) those in which food quantities are recalled by tools such as household measures, food models, food replicas and food photographs. Also standard portions are used frequently.

The most accurate method for measuring food intake is weighing foods before and after eating ${ }^{(2)}$. This method is not always the most appropriate however, because it imposes a large burden on participants, is expensive and time consuming, and does not allow assessment of past intakes ${ }^{(2)}$.

Visual aids, such as food photographs, help circumvent many of these problems and may help improve the accuracy of food portion quantification ${ }^{(3-7)}$. However, there is a crucial need to determine the error level induced by such an approach ${ }^{(8-13)}$.

Some studies have evaluated the errors related to perception of foods in photographs while assessing the portion of foods presented to the participant ${ }^{(3,6-8,13,14)}$. They speculate that assessment of portion size would not be biased by factors such as participants' unwillingness to divulge what they have eaten ${ }^{(1,15)}$. Others have estimated the error arising from conceptualization of foods and memory while recalling amounts eaten by the participant $^{(4,5,16,17)}$. They consider that the effectiveness of portion size estimation may be reduced when memory/ recall is required ${ }^{(7,8)}$.

The need to assess the rapid changes in dietary habits in the Mediterranean area over recent years and the lack of data in Lebanon urged us to perform dietary surveys in this country. To fulfil this objective, a first task consisted in developing a photographic atlas to quantify portion sizes of 212 traditional Lebanese dishes, presently not available. 
The present study describes the methodology used to design and validate this colour photographic atlas. Discrepancies between the food portion size deduced from the photographs and the corresponding real portion weight were assessed and main bias identified.

Specifically, we evaluated (i) the errors related to perception of foods in photographs while assessing the amounts of foods presented to participants (study 1) and (ii) the errors related to perception, conceptualization of foods and memory while recalling amounts eaten by participants on the previous day (study 2).

\section{Methods}

\section{Design of the photographic atlas}

\section{Choice of dishes}

The photographic atlas presents 633 colour photographs (format $7.6 \mathrm{~cm} \times 4.7 \mathrm{~cm}$ ) corresponding to 212 traditional dishes, usually consumed at home and in Lebanese restaurants, and cooked according to two main Lebanese recipe books ${ }^{(18,19)}$.

\section{Portion sizes}

The dishes appear according to three portion sizes presented on the same conventional plate. Each portion is associated with a letter. The general principle is to identify the consumed portion, either in one of the suggested photographs (B, D or F) or between two consecutive photographs ( $\mathrm{C}$ or $\mathrm{E}$ ), including below or beyond the extreme photographs (A or G; Fig. 1).

\section{Portion weights}

The medium portion (D) was chosen in a way to be representative of the mean portion size actually consumed by the Lebanese population, as deduced from a preliminary food consumption survey carried out between March and June 2004 on fifty Lebanese adult volunteers (twenty-five men and twenty-five women) in which portion sizes of 212 dishes were estimated by household measures (M Tueni and A Mounayar, unpublished results).

For the majority of the dishes, we added to or subtracted from the weight of the medium portion a coefficient (2SD) for the small (B) and the large (F) portions, a coefficient (SD) for the intermediate virtual portions (C and E) and a coefficient (3SD) for the extreme virtual portions (A and $G$ ):

$$
\begin{gathered}
\mathrm{X}-3 \mathrm{SD}(\mathrm{A}) ; \mathrm{X}-2 \mathrm{SD}(\mathrm{B}) ; \mathrm{X}-\mathrm{SD}(\mathrm{C}) ; \mathrm{X}(\mathrm{D}) ; \\
\mathrm{X}+\mathrm{SD}(\mathrm{E}) ; \mathrm{X}+2 \mathrm{SD}(\mathrm{F}) ; \mathrm{X}+3 \mathrm{SD}(\mathrm{G})
\end{gathered}
$$

where $\mathrm{X}$ is the weight of the medium portion (D). The SD coefficient represented the standard deviation of the mean consumption for each food. In order to select portions neither too small nor too large, some food photographs depicting different portions, computed as described above, were shown to the fifty Lebanese adult volunteers and we asked them their opinion. For example, the mean consumption of mdardara was 200 (SD 45) g, which results in a portion $\mathrm{B}$ of $110 \mathrm{~g}$ and a portion $\mathrm{F}$ of $290 \mathrm{~g}$. According to the volunteers' opinions, we decreased $\mathrm{B}$ to $100 \mathrm{~g}$ and increased $\mathrm{F}$ to $300 \mathrm{~g}$. Thus, the (SD) coefficient will be 50 (Fig. 1).

\section{Food digital photographs}

Three different portions of each dish were weighed accurately (Tefal 3000 digital electronic scale, $\pm 1 \mathrm{~g}$ precision), placed on a white dining plate contrasting with a colour background and photographed from the same angle using a digital camera (Sony-Cyber 72 Power Shot).

\section{Validation of the photographic atlas}

Two groups of Lebanese volunteers validated the photographic atlas. They were recruited by telephone; the phone numbers were selected randomly from the phone book. The study was approved by the Ethical Committee of the Lebanese University.

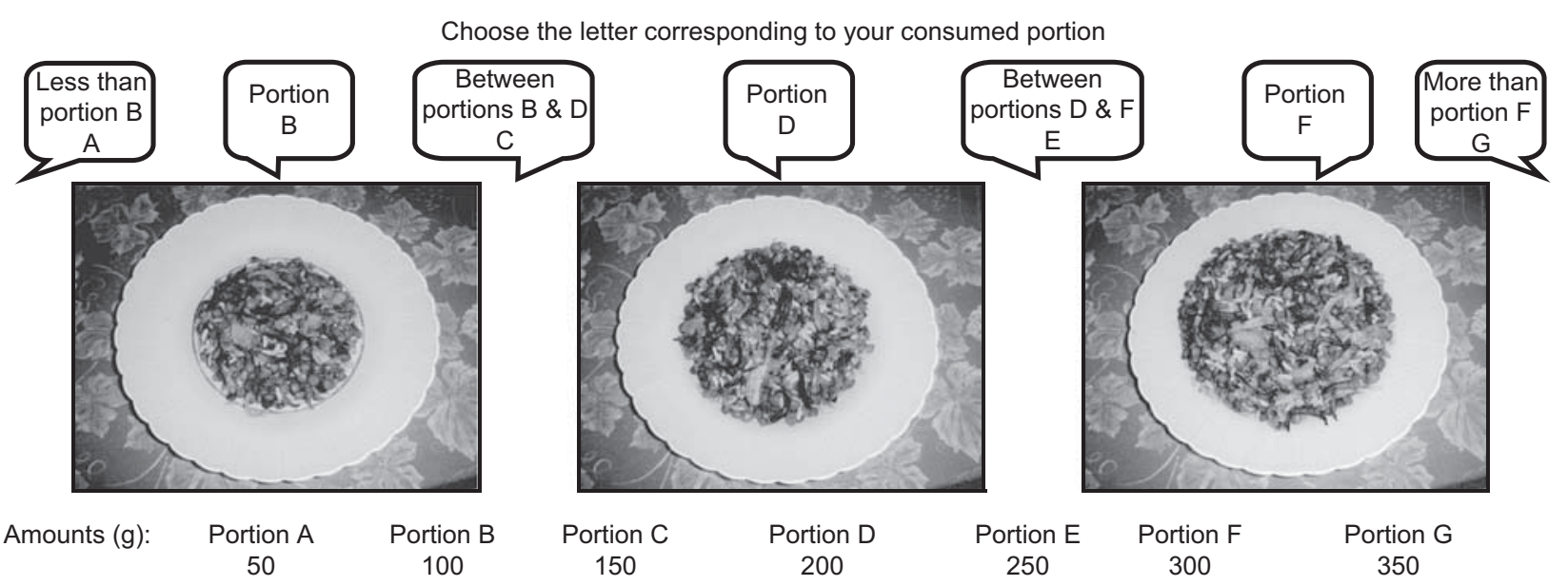

Fig. 1 The set of pictures of mdardara (lentils with rice) in three portion sizes and the amounts in grams 
Table 1 Food groups, shapes and Lebanese traditional dishes included in the present study

\begin{tabular}{|c|c|}
\hline Food category/shape & Lebanese traditional dish \\
\hline Meat/number and size of pieces & $\begin{array}{l}\text { Kafta mechwieh (pieces of ground meat and chopped parsley), chich taouk (pieces of } \\
\text { broiled chicken), lahmeh mechwieh (pieces of broiled meat), kebbe bil saniyeh } \\
\text { (pieces of ground meat and bulgur) }\end{array}$ \\
\hline Vegetables/area and depth of mounds on plate & $\begin{array}{l}\text { Tabbouleh (parsley, tomatoes and bulgur salad), fattouch (fresh vegetable salad with } \\
\text { bread), salatet baakle (purslane salad), salatet roka (arugula salad), salatet zaatar } \\
\text { (thyme salad), m'saquaa (aubergine salad), baba ghannouj (mashed aubergine with } \\
\text { sesame butter), silk (beet salad), hindbeh be zeit (chicory and fried onions) }\end{array}$ \\
\hline $\begin{array}{l}\text { Vegetables/area and depth of foods in sauce or } \\
\text { gravy spreading across plate }\end{array}$ & $\begin{array}{l}\text { Bemieh (okra in oil), loubieh bil zeit (green beans in oil), yachnet bazella (pea stew), } \\
\text { yachnet sabanegh (spinach stew), yachnet loubieh (green bean stew), molokhieh } \\
\text { (Jew's mallow stew) }\end{array}$ \\
\hline Vegetables/number and size of pieces & $\begin{array}{l}\text { Batinjane meqli (fried aubergine), warak aanab (stuffed grape leaves), koussa mehchi } \\
\text { (stuffed courgettes) }\end{array}$ \\
\hline $\begin{array}{l}\text { Legumes/area and depth of foods in sauce or } \\
\text { gravy spreading across plate }\end{array}$ & Adass be hamoud (lentil, beet and potato soup) \\
\hline Legumes/area and depth of mounds on plate & $\begin{array}{l}\text { Mjaddara (mashed lentils and rice), mdardara (lentils and rice), hommos be thineh } \\
\text { (mashed chickpeas with sesame butter), hommos hab (chickpea salad), foul } \\
\text { mdammas (fava bean salad) }\end{array}$ \\
\hline Legumes/number and size of pieces & Falafel (chickpea and broad bean croquettes) \\
\hline Cereals/area and depth of mounds on plate & Riz mfalfal (rice pilaf), riz aa djej (rice with chicken) \\
\hline $\begin{array}{l}\text { Cereals/area and depth of foods in sauce or } \\
\text { gravy spreading across plate }\end{array}$ & $\begin{array}{l}\text { Chich barak (ravioli with cooked yoghurt), maacaronis bil saniyeh (pasta with tomato } \\
\text { sauce), borghol be dfine (bulgur with meat and sauce) }\end{array}$ \\
\hline Cereals and bread/number and size of pieces & $\begin{array}{l}\text { Rkakat be jibne (cheese rolls), samboussik (meat rissoles), fatayer be sbanegh } \\
\text { (spinach turnover), fatayer be jibne (cheese turnover) }\end{array}$ \\
\hline $\begin{array}{l}\text { Cereals and bread/area and depth of wedge } \\
\text { Sweets/number and size of pieces }\end{array}$ & $\begin{array}{l}\text { Manakish (thyme pie), lahmeh baajin (meat pie), khebez arabeh (Lebanese bread, pita) } \\
\text { Graybeh (Lebanese cookies), kol w shkor (pastry with almonds), beklawa (pastry with } \\
\text { pine nuts), sfouf (pine nut cake), nammoura (wheatmeal cookies with almonds), } \\
\text { seniora (Lebanese cookies) }\end{array}$ \\
\hline
\end{tabular}

Study 1: Validation of presented portion size using food photographs

Fifty adults were invited to the Lebanese university kitchen but forty adults (twenty males, twenty females) completed the first protocol in May 2006. The nonresponse rate was $20 \%$.

The ten foods selected (kafta mechwieh, kebbe bil saniyeh, koussa mebchi, loubieb bil zeit, mjaddara, riz aa djej, samboussik, waraak aanab, tabbouleh, yachnet bazella) were the most consumed Lebanese dishes based on the preliminary survey described above. Participants were shown one portion size for each of the ten foods, accurately pre-weighed (Tefal 3000 digital electronic scale), and asked by an interviewer to compare the presented food with the photographs.

Study 2: Validation of previous day's portion size using food photographs

Sixty-five adults were invited but fifty adults (twenty-five males, twenty-five females) completed the second protocol between February and April 2007. The non-response rate was $23 \%$.

Each participant weighed all foods consumed (Table 1) during one meal at home (Tefal 3000 digital electronic scale) and the next day recalled the amount of food that was eaten the previous day using the photographic atlas.

\section{Analysis and treatment of results}

The amounts of food presented or eaten and estimated by the photographs were compared. The differences were calculated and expressed as percentages of the weights of food presented or consumed. Thus, a positive value indicates an overestimate of the weight and a negative value an underestimate. Differences between estimated and actual quantities were assessed using a signed rank test. The distribution patterns of individual errors were described according to the $25 \mathrm{th}, 50$ th and 75 th percentiles of total error variability for a given food. Spearman's correlation coefficients were calculated in order to evaluate the extent of the linear relationship between amounts estimated by photographs and amounts presented or consumed. Agreement between the methods was evaluated according to Bland and Altman ${ }^{(20)}$.

The SPSS for Windows statistical software package version 10·1 (2001; SPSS Inc., Chicago, IL, USA) and Analyse-it ${ }^{\circledR}$ statistical software for Microsoft Excel ${ }^{\circledR}$ (Analyse-it Software Ltd, Leeds, UK) were used for statistical analyses.

\section{Results}

Forty participants performed 400 estimations, twenty-two were aged 21-41 years and eighteen were aged 42-62 years (study 1). Fifty participants performed 182 estimations, twenty-six were 21-41 years old and twenty-four were 42-62 years old (study 2). In study 1 five foods were overestimated (range from $+0.9 \%$ for samboussik to $+24.5 \%$ for loubieh bil zeit) and five were underestimated (range from $-3 \cdot 6 \%$ for tabbouleb to $-13 \cdot 1 \%$ 
for kafta mechwieb). The amounts of food eaten (mean and SD) and subsequently estimated with photographs are presented in Table 2 (study 2). Two categories of food were overestimated (range from $3 \cdot 4 \%$ for legumes to $3.8 \%$ for meat) and three were underestimated (range from $-2 \cdot 7 \%$ for vegetables to $-10 \cdot 4 \%$ for cereals). Foods served in sauce or gravy (error $+14.7 \%$ ) and wedges (error $+1 \cdot 3 \%$ ) were overestimated but food served in pieces of different size (error $-12 \cdot 1 \%$ ) and mounds (error $-8 \cdot 7 \%)$ were underestimated. The portion size of loubieh bil zeit, tabbouleh and yachnet bazella estimated with photographs was significantly different from the real amount presented (signed rank test: $P<0.001, P=0.04$ and $P=0.004$, respectively). For all categories of food there was no significant difference between the amounts consumed and those estimated by photographs. The distributions of the errors for each food category are included in Table 2 . Errors above $+100 \%$ only concerned warak aanab, kafta mechwieh, legumes and foods served in sauce or gravy and mounds.

The difference of large portion sizes was $+1.5 \%$ and small portion sizes $-18 \cdot 2 \%$.

Bland-Altman plots showed good agreement for all dishes, with a negligible bias of $-0 \cdot 2 \mathrm{~g}$ between estimated and presented portions, and $-6.3 \mathrm{~g}$ between estimated and eaten portions.

Spearman correlation coefficients suggested that estimated portions with photographs were closely ranked with presented portions $(r=0 \cdot 88, P<0 \cdot 01)$ or consumed portions $(r=0 \cdot 85, P<0 \cdot 01)$ for all foods. However, the Spearman correlation coefficient was lower for yachnet bazella (0.57), tabbouleh (0.57), riz aa djej (0.59), meat $(0.56)$ and legumes (0.57).

Comparison between errors for the ten foods used in study 1 and the same foods in study 2 showed that errors increased slightly from $-0 \cdot 1 \%$ to $+3 \cdot 24 \%$.

\section{Discussion}

In the present study, we evaluated the mean error specifically associated with the perception of food while presenting the food without consuming. In addition, the mean error due to conceptualization and memory was assessed by estimating the portion size of food eaten the day before. The first approach is less realistic than the second one which is representative of a $24 \mathrm{~h}$ recall situation, but has the advantage of allowing a higher number of foods to be tested in one session (400 evaluations) and enabling a more accurate quantification of the error between estimation and weight. Participants of the second group weighed their own food at home before and after consumption. The situation is more representative of the dietary questionnaire situation, where volunteers eat in their usual environment, but with the common bias of the higher attention inappropriately paid 
to the portion size they consume. In addition, a probable error could have been introduced during weighing or recording of the food portion.

Extreme differences between actual and estimated portion sizes using photographs ranged from $-13 \cdot 1 \%$ for the dish kafta mechwieh to $+24 \cdot 5 \%$ for the dish loubieh bil zeit, and from $-10.4 \%$ for cereals and bread to $+3.8 \%$ for meat. Except for the dishes loubieh bil zeit, tabbouleb and yachnet bazella for which errors were found suggesting perception problems, differences were rather small and not significant, thus validating that the photographic atlas is a reliable way to estimate food portion size. Huybregts et $a l^{(21)}$ found that the mean differences between served and estimated portion sizes were between $-8 \cdot 4 \%$ for couscous and $+6 \cdot 3 \%$ for liquid sauce, while Turconi et $a l^{(17)}$ found that the mean differences between estimated and actual portion weights were between $-2 \cdot 7 \%$ for bread and $+15 \cdot 9 \%$ for vegetables.

The participants in our study had more difficulty in quantifying the dish kafta mechwieh (group level error $-13 \cdot 1 \%$; range $-56 \cdot 2 \%$ to $+110 \%$ ), whereas the dish koussa mehchi was quantified relatively accurately (group level error $+3 \cdot 3 \%$; range $-5 \cdot 5 \%$ to $+39 \cdot 3 \%$ ). The fact that some foods appear to be more difficult to estimate accurately than others is a common finding. Robson and Livingstone $^{(15)}$ found that cheese was the most difficult to estimate (range $-38.9 \%$ to $+284.6 \%$ ), while orange juice was relatively easy (range $-21.5 \%$ to $+34.6 \%$ ).

In the present study, foods served in sauce or gravy and in pieces of different size were more difficult to estimate than foods served in wedges or mounds. Similarly, the participants in the study of Nelson et $a l^{(5)}$ had no difficulty in estimating wedges of quiche or mounds of boiled rice.

Other studies ${ }^{(3,5)}$ have shown the flat slope phenomenon; small portion sizes are overestimated and large portion sizes are underestimated. However, we could not observe this trend in our data. The consistency between portion weights and estimated portion sizes of foods presented or eaten is revealed by high mean correlation coefficients $(0.88$ for individual foods and 0.85 for different food categories). Haraldsdottir et al. ${ }^{(22)}$ found Pearson correlation coefficients between 0.22 and 0.54 , while a mean correlation coefficient of 0.82 was obtained by Turconi et $a l^{(17)}$.

In the present work, the errors and bias in study 2 were larger than those in study 1 . This difference in accuracy seems to illustrate that the effectiveness of this method may be slightly reduced when memory recall is required. Other studies ${ }^{(15,16)}$ have also noted that.

\section{Conclusions}

The colour photographic atlas developed in the present study appears to be a useful tool for portion size estimation of the most common traditional Lebanese dishes.

\section{Acknowledgements}

This research received no specific grant from any funding agency in the public, commercial or not-for-profit sector. The authors declare that they have no conflicts of interest. M.T. was involved in the conception and design of the study, collected the data, performed the analysis and interpretation and wrote the paper. A.M. was involved in the conception and design of the study and collected the data. I.B.-A. was involved in the conception and design of the study and made a contribution in drafting of the paper. The authors thank the volunteers who took part in the study.

\section{References}

1. Howat PM, Mohan R, Champagne C et al. (1994) Validity and reliability of reported dietary intake data. J Am Diet Assoc 94, 169-173.

2. Wolper C, Heshka S \& Heymsfield SB (1995) Measuring food intake: an overview. In Handbook of Assessment Measures for Eating Behaviours and Weight-Related Problems, pp. 215-240 [D Allison, editor]. Thousand Oaks, CA: Sage Publishing

3. Nelson M, Atkinson M \& Darbyshire S (1994) Food photography I: the perception of food portion size from photographs. Br J Nutr 72, 649-663.

4. Nelson M \& Haraldsdottir J (1998) Food photographs: practical guidelines I. Design and analysis of studies to validate portion size estimates. Public Health Nutr 1, 219-230.

5. Nelson M, Atkinson M \& Darbyshire S (1996) Food photography II: use of food photographs for estimating portion size and the nutrient content of meals. BrJ Nutr 76, 31-49.

6. Robinson F, Morritz W, McGuinness P et al. (1997) A study of the use of a photographic food atlas to estimate served and self-served portion sizes. J Hum Nutr Diet 10, 117-124.

7. Frobisher C \& Maxwell SM (2003) The estimation of food portion sizes: a comparison between using descriptions of portion sizes and a photographic food atlas by children and adults. J Hum Nutr Diet 16, 181-188.

8. Lucas F, Niravong M, Villeminot S et al. (1995) Estimation of food portion size using photographs: validity, strengths, weakness and recommendations. J Hum Nutr Diet 8, 65-74.

9. Navarro A, Cristaldo PE, Diaz MP et al. (2000) Food photography atlas: its suitability for quantifying food and nutrient consumption in nutritional epidemiological research in Cordoba, Argentina. Rev Fac Cien Med Univ Nac Cordoba 57, 67-74.

10. Chambers IV E, McGuire B, Godwin S et al. (2000) Quantifying portion sizes for selected snacks and beverages in 24-h dietary recalls. Nutr Res 20, 315-326.

11. Godwin SL, Chambers ET \& Cleveland L (2004) Accuracy of reporting dietary intake using various portion-size aids in-person and via telephone. J Am Diet Assoc 104, 585-594.

12. Foster E, Matthews JN, Nelson M et al. (2006) Accuracy of estimates of food portion size using food photographs the importance of using age-appropriate tools. Public Health Nutr 9, 509-514.

13. Hernandez T, Wilder L, Kuehn D et al. (2006) Portion size estimation and expectation of accuracy. J Food Compost Anal 19, S14-S21. 
14. Ovaskainen ML, Paturi M, Reinivuo $\mathrm{H}$ et al. (2008) Accuracy in the estimation of food servings against the portions in food photographs. Eur J Clin Nutr 62, 674-681.

15. Robson PJ \& Livingstone MBE (1999) An evaluation of food photographs as a tool for quantifying food and nutrient intakes. Public Health Nutr 3, 183-192.

16. Faggiano F, Vineis P, Cravanzola D et al. (1992) Validation of a method for the estimation of food portion size. Epidemiology 3, 379-382.

17. Turconi G, Guarcello M, Gigli Berzolari F et al. (2005) An evaluation of a color food photography atlas as a tool for quantifying food portion size in epidemiological dietary surveys. Eur J Clin Nutr 59, 923-931.
18. El Haje A (2000) Chef Antoine maakoul el hana. Beirut: Charles Mouannes Printing.

19. Shwayri R (1998) Chef Ramzi fi aalam el sabah. Beirut: Academia International Publishing.

20. Bland JM \& Altman DG (1986) Statistical methods for assessing agreement between two methods of clinical measurement. Lancet 1, 307-310.

21. Huybregts L, Roberfroid D, Lachat C et al. (2008) Validity of photographs for food portion estimation in a rural West African setting. Public Health Nutr 11, 581-587.

22. Haraldsdottir J, Tjonneland A \& Overvad K (1994) Validity of individual portion size estimates in a food frequency questionnaire. Int J Epidemiol 23, 786-796. 